\title{
Multi-centered first order formalism
}

\author{
Sergio Ferrara, ${ }^{a, c}$ Alessio Marrani, ${ }^{a}$ Andrey Shcherbakov ${ }^{c}$ and Armen Yeranyan ${ }^{b, c, d}$ \\ ${ }^{a}$ Physics Department, Theory Unit, CERN, \\ CH 1211, Geneva 23, Switzerland \\ ${ }^{b}$ Museo Storico della Fisica e Centro Studi e Ricerche "Enrico Fermi", \\ Via Panisperna 89A, I-00184 Roma, Italy \\ ${ }^{c}$ INFN - Laboratori Nazionali di Frascati, \\ Via Enrico Fermi 40, I-00044 Frascati, Italy \\ ${ }^{d}$ Department of Physics, Yerevan State University, \\ Alex Manoogian St. 1, Yerevan, 0025, Armenia \\ E-mail: sergio.ferrara@cern.ch, alessio.marrani@cern.ch, \\ ashcherb@lnf.infn.it, ayeran@lnf.infn.it
}

ABSTRACT: We propose a first order formalism for multi-centered black holes with flat three-dimensional base-space, within the stu model of $N=2, D=4$ ungauged MaxwellEinstein supergravity. This provides a unified description of first order flows of this universal sector of all models with a symmetric scalar manifold which can be obtained by dimensional reduction from five dimensions.

We develop a $D=3$ Cartesian formalism which suitably extends the definition of central and matter charges, as well as of black hole effective potential and first order "fake" superpotential, in order to deal with not necessarily axisimmetric solutions, and thus with multi-centered and/or (under-)rotating extremal black holes.

We derive general first order flow equations for composite non-BPS and almost BPS classes, and we analyze some of their solutions, retrieving various single-centered (static or under-rotating) and multi-centered known systems.

As in the $t^{3}$ model, the almost BPS class turns out to split into two general branches, and the well known almost BPS system is shown to be a particular solution of the second branch.

Keywords: Black Holes in String Theory, Black Holes, Supergravity Models

ArXiv ePrint: 1211.3262 


\section{Contents}

1 Introduction 1

2 Second order equations of motion 3

$\begin{array}{lll}3 & \text { First order equation of motion } & 7\end{array}$

3.1 Construction of flows 8

3.1.1 BPS

$\begin{array}{lll}3.1 .2 & \text { Almost BPS } & 10\end{array}$

$\begin{array}{lll}3.1 .3 & \text { Composite non-BPS } & 12\end{array}$

4 Composite non-BPS class 12

$\begin{array}{ll}4.1 \text { Single-centered solutions } & 13\end{array}$

$\begin{array}{lll}4.2 & \text { Multi-centered solutions } & 15\end{array}$

$\begin{array}{lll}\text { 4.2.1 Electric configuration } & 15\end{array}$

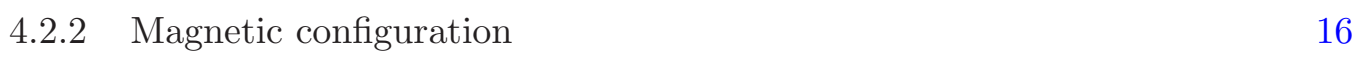

5 Almost BPS class $\quad 17$

$\begin{array}{lll}5.1 & \text { Branch I } & 19\end{array}$

$\begin{array}{lll}5.2 & \text { Branch II } & 20\end{array}$

$\begin{array}{llr}6 & \text { Conclusion } & 21\end{array}$

$\begin{array}{ll}\text { A Notations and useful formulæ } & 23\end{array}$

\section{Introduction}

Multi-centered extremal black hole (BH) solutions in four-dimensional supergravity theories have been widely investigated in recent years [1-15].

Motivated by the issue of matching the true BPS spectrum and the spectrum of spherically symmetric BHs in supergravity, in [16] a supersymmetric class of multi-centered BHs was introduced, whose BPS first order flow equations were then solved and analyzed in [17], revealing interesting features, such as fixed distance among the centers (in presence of mutually non-local electric-magnetic charge vectors).

However, only in the last two years a group-theoretical approach [1,2], based on nilpotent orbits and timelike reduction to three dimensions, allowed for a systematic construction and investigation of whole new classes of solutions, in which some or all $\mathrm{BH}$ centers are non-supersymmetric (non-BPS).

An elegant approach to the flow dynamics of scalar fields in the background of singlecentered extremal BH solutions of Maxwell-Einstein theories of (super)gravity, essentially 
based on the first order reformulation of the scalar equations of motion, was introduced in [18], and then developed in various works [19-24]. The possibility to switch from second order to first order differential equations of motion - without doubling their number - has an applicative relevance. Indeed, due to the interplay between auxiliary fields and scalar charges, the first order formalism automatically discards blowing-up solutions; furthermore, the integration of first order equations is surely more manageable, and explicit forms of attractor flows can be more easily determined.

The extension of such a formalism to non-supersymmetric multi-centered configurations was started in $[14,15]$ by reducing the relevant action to a sum of squares, however without yielding explicit expressions for the flow equations and their corresponding governing functions. A consistent and explicit determination of the most general first order flow equations for non-BPS multi-centered and/or rotating BHs with flat three-dimensional base-space was achieved in [25], within the simplest model of $N=2, D=4$ MaxwellEinstein ungauged supergravity with a cubic prepotential, namely the so-called $t^{3}$ model, exhibiting only one vector multiplet, and whose uplift to $D=5$ is "pure" minimal supergravity (see e.g. [26]).

In the present investigation, we further develop the approach of [25], and determine the general first order flow equations for all classes of multi-centered $\mathrm{BH}$ solutions with flat three-dimensional base-space in the so-called stu model [27, 28]; in this model, three vector multiplets are coupled, in a triality-invariant way, to the $N=2$ gravity multiplet, and the resulting completely factorized rank-3 symmetric special Kähler manifold $[\mathrm{SU}(1,1) / \mathrm{U}(1)]^{3}$ can be considered a universal sector of all symmetric scalar manifolds of $D=4, N \geqslant 2$ extended supergravity theories which admit a $D=5$ uplift.

A key result is the reformulation of second order equations of motion in a manifestly $D=3$ Cartesian formalism, based on a timelike Lagrangian reduction $D=4 \rightarrow 3$ in a stationary BH background [29], later specialized for flat spatial slices. This formalism allows for a consistent generalization of the BH effective potential [30], and of its expression in terms of supersymmetry central charges and matter charges, as well as in terms of a first order "fake" superpotential [18], in not necessarily axisymmetric contexts, which thus can include multi-centered solutions.

Various under-rotating stationary (BPS and non-BPS) single-centered solutions, as well as the known classes of $B P S[16]$, composite non-BPS [1] and almost BPS [1, 3] multi-centered solutions (possibly constrained in a suitable way) are retrieved as particular solutions of the various first order systems which we propose. It should be stressed that, analogously to [25], in this paper we focus on the general formulation of first order systems of equations, leaving the determination of new sets of solutions and the investigation of their physical properties to future investigations.

The paper is organized as follows.

In section 2, we start with the timelike reduction of Maxwell-Einstein-scalar action in the background of stationary metrics (then assumed to have flat spatial slices). As mentioned, this yields to a $D=3$ Cartesian formalism, which is especially suitable to handle multi-centered and under-rotating single-centered solutions. 
Our general approach to first order formalism is then exploited in section 3 for the various classes of multi-centered solutions, namely the BPS (section 3.1.1), almost BPS (section 3.1.2) and composite non-BPS (section 3.1.3) classes, also retrieving and discussing known multi- and single- centered solutions. In particular, as found in [25] in the $t^{3}$ model, we find that the almost BPS class splits into two branches, which can essentially be related to the BPS or non-BPS nature of the corresponding single-centered limits, as discussed in sections 5.1 and 5.2 .

Finally, section 6 contains a summary and an outlook of results.

Some notation and useful formulæ are given in appendix A.

\section{Second order equations of motion}

Let us consider the $D=4$ Einstein-Maxwell action

$$
S=\int d^{4} x \sqrt{-g}\left[-\frac{1}{2} R+G_{a \bar{a}} g^{\mu \nu} \partial_{\mu} z^{a} \partial_{\nu} \bar{z}^{\bar{a}}+\frac{1}{4} \mu_{\Lambda \Sigma} F_{\mu \nu}^{\Lambda} F^{\Sigma \mu \nu}+\frac{1}{4} \nu_{\Lambda \Sigma} F_{\mu \nu}^{\Lambda *} F^{\Sigma \mu \nu}\right],
$$

which, depending on properties of the target space metric $G_{a \bar{b}}(z, \bar{z})$ and of the coupling matrices $\mu_{\Lambda \Sigma}(z, \bar{z})$ and $\nu_{\Lambda \Sigma}(z, \bar{z})$, may be the bosonic sector of some $N \geqslant 2$-extended, $D=4$ Maxwell-Einstein supergravity. In the present investigation, we are interested in stationary solutions to the Einstein-Maxwell equations of motion; we will thus perform a timelike reduction to tree dimensions à la Breitenlohner-Gibbons-Maison [29], giving rise to a $D=3$ Cartesian formalism, which is not necessarily axisymmetric.

We start from a space-time metric satisfying the following $\operatorname{Ansatz}(i, j=1,2,3)$ :

$$
d s^{2}=g_{\mu \nu} d x^{\mu} d x^{\nu}=e^{2 \mathrm{U}(x)}\left(d t+\omega_{i}(x) d x^{i}\right)^{2}-e^{-2 \mathrm{U}(x)} \gamma_{i j}(x) d x^{i} d x^{j} .
$$

In this background, the Abelian vector potential $A_{\mu}^{\Lambda}$ (defining the two-form field strength ${ }^{1}$ $\left.F_{\mu \nu}^{\Lambda}=2 \partial_{[\mu} A_{\nu]}^{\Lambda}\right)$ splits into $D=3$ vector potentials $a_{i}^{\Lambda}$ and Kaluza-Klein scalars $b^{\Lambda}$ :

$$
A_{\mu}^{\Lambda} d x^{\mu}=b^{\Lambda}\left(d t+\omega_{i} d x^{i}\right)+a_{i}^{\Lambda} d x^{i} .
$$

By using the $D=3$ vectors $a_{i}^{\Lambda}$ and $\omega_{i}$, one can construct the corresponding field strengths

$$
f_{i j}^{\Lambda}=2 \partial_{[i} a_{j]}^{\Lambda}, \quad W_{i j}=2 \partial_{[i} \omega_{j]},
$$

respectively expressing the magnetic field and the rotation in tree dimensions.

On stationary background (2.2), the action (2.1) is equivalent to the following $D=3$ one:

$$
\begin{aligned}
S=\int d^{3} x \sqrt{\gamma}[- & \frac{1}{2} R^{(3)}+G_{a \bar{a}} \gamma^{i j} \partial_{i} z^{a} \partial_{j} \bar{z}^{\bar{a}}+\gamma^{i j} \partial_{i} U \partial_{j} U-\frac{1}{8} e^{4 U} W_{i j} W^{i j} \\
& +\frac{1}{2} e^{-2 U} \mu_{\Lambda \Sigma} \gamma^{i j} \partial_{i} b^{\Lambda} \partial_{j} b^{\Sigma}-\frac{1}{4} e^{2 U} \mu_{\Lambda \Sigma}\left(f_{i j}^{\Lambda}+b^{\Lambda} W_{i j}\right)\left(f^{\Sigma i j}+b^{\Sigma} W^{i j}\right) \\
& \left.-\nu_{\Lambda \Sigma} \partial_{i} b^{\Lambda}\left({ }^{*} f^{\Sigma i}+b^{\Sigma}{ }^{*} W^{i}\right)\right]
\end{aligned}
$$

\footnotetext{
${ }^{1}$ Throughout the paper, the (anti)symmetrization is defined with a $1 / n$ ! normalization if applied to $n$ indices.
} 
where the $D=3$ Hodge operator " $*$ " is defined with respect to the 3 -dimensional metric $\gamma_{i j}$ :

$$
{ }^{*} A^{i} \equiv \frac{1}{2 \sqrt{\gamma}} \varepsilon^{i j k} A_{j k}
$$

The $D=3$ two-forms $f_{i j}^{\Lambda}$ and $W_{i j}$ can be dualized into scalars $b_{\Lambda}$ and $\psi$ by adding the following terms to the action (2.3)

$$
\int d^{3} x\left[\frac{1}{2} b_{\Lambda} \epsilon^{i j k} \partial_{i} f_{j k}^{\Lambda}+\frac{1}{2} \psi \epsilon^{i j k} \partial_{i} W_{j k}\right]
$$

thus allowing for the two-forms to be expressed in terms of their duals:

$$
\begin{aligned}
f^{\Lambda i j}+b^{\Lambda} W^{i j} & =-\frac{1}{\sqrt{\gamma}} e^{-2 U} \mu^{\Lambda \Sigma} \varepsilon^{i j k}\left(\partial_{k} b_{\Sigma}+\nu_{\Sigma \Sigma^{\prime}} \partial_{k} b^{\Sigma^{\prime}}\right) \\
W^{i j} & =-\frac{2}{\sqrt{\gamma}} e^{-4 U} \varepsilon^{i j k}\left(\partial_{k} \psi-b^{\Lambda} \partial_{k} b_{\Lambda}\right),
\end{aligned}
$$

as well as the action (2.3)to be recast as:

$$
\begin{aligned}
S=\int d^{3} x \sqrt{\gamma}[ & -\frac{1}{2} R^{(3)}+G_{a \bar{a}} \gamma^{i j} \partial_{i} z^{a} \partial_{j} \bar{z}^{\bar{a}}+\gamma^{i j} \partial_{i} U \partial_{j} U+\frac{1}{2} e^{-2 U} \mu_{\Lambda \Sigma} \gamma^{i j} \partial_{i} b^{\Lambda} \partial_{j} b^{\Sigma} \\
& +\frac{1}{2} e^{-2 U} \mu^{\Lambda \Sigma} \gamma^{i j}\left(\partial_{i} b_{\Lambda}+\nu_{\Lambda \Lambda^{\prime}} \partial_{i} b^{\Lambda^{\prime}}\right)\left(\partial_{j} b_{\Sigma}+\nu_{\Sigma \Sigma^{\prime}} \partial_{j} b^{\Sigma^{\prime}}\right) \\
& \left.+e^{-4 U} \gamma^{i j}\left(\partial_{i} \psi-b^{\Lambda} \partial_{i} b_{\Lambda}\right)\left(\partial_{j} \psi-b^{\Sigma} \partial_{j} b_{\Sigma}\right)\right] .
\end{aligned}
$$

By grouping the Kaluza-Klein scalars $b^{\Lambda}$ and the scalars $b_{\Lambda}$ (dual to $f_{i j}^{\Lambda}$ ) into a symplectic vector $b^{\alpha}$ (with $\alpha$ running over contravariant and covariant symplectic index $\Lambda$ ) and introducing a scalar $\tilde{\psi}$

$$
b^{\alpha} \equiv\left(\begin{array}{c}
b^{\Lambda} \\
b_{\Lambda}
\end{array}\right), \quad \tilde{\psi} \equiv 2 \psi-b^{\Lambda} b_{\Lambda},
$$

the action (2.5) can be rewritten in more compact form:

$$
\begin{aligned}
S=\int d^{3} x \sqrt{\gamma}[ & -\frac{1}{2} R^{(3)}+G_{a \bar{a}} \gamma^{i j} \partial_{i} z^{a} \partial_{j} \bar{z}^{\bar{a}}+\gamma^{i j} \partial_{i} U \partial_{j} U+\frac{1}{2} e^{-2 U} \gamma^{i j} \partial_{i} b^{\alpha} M_{\alpha \beta} \partial_{j} b^{\beta} \\
& \left.+\frac{1}{4} e^{-4 U} \gamma^{i j}\left(\partial_{i} \tilde{\psi}+\left\langle b, \partial_{i} b\right\rangle\right)\left(\partial_{j} \tilde{\psi}+\left\langle b, \partial_{j} b\right\rangle\right)\right]
\end{aligned}
$$

For the definitions of the symplectic symmetric matrix $M_{\alpha \beta}$ and the skew-symmetric symplectic product $\langle\cdot, \cdot\rangle$, see appendix A. Then, by introducing a symplectic vector $f_{i j}^{\alpha}=$ $\left(f_{i j}^{\Lambda}, f_{\Lambda i j}\right)^{\top}$ constructed from the field strengths, eqs. (2.4) enjoy a manifest symplectic covariance: ${ }^{2}$

$$
\begin{aligned}
f_{i j}^{\alpha} & =\sqrt{\gamma} \epsilon_{i j k} \gamma^{k k^{\prime}} e^{-2 U} M^{\alpha \beta} \Omega_{\beta \gamma} \partial_{k^{\prime}} b^{\gamma}-b^{\alpha} W_{i j} ; \\
W_{i j} & =-\sqrt{\gamma} e^{-4 U} \epsilon_{i j k} \gamma^{k l}\left(\partial_{l} \tilde{\psi}+\left\langle b, \partial_{l} b\right\rangle\right) .
\end{aligned}
$$

\footnotetext{
${ }^{2}$ Eq. (2.4a) is just the upper half of eq. (2.8a).
} 
The second order equations of motion pertaining to the action (2.7) consist of the Einstein equations

$$
\begin{aligned}
\frac{1}{2} R_{i j}^{(3)}= & G_{a \bar{a}} \partial_{(i} z^{a} \partial_{j)} \bar{z}^{\bar{a}}+\partial_{i} U \partial_{j} U+\frac{1}{2} e^{-2 U} \partial_{i} b^{\alpha} M_{\alpha \beta} \partial_{j} b^{\beta} \\
& +\frac{1}{4} e^{-4 U}\left(\partial_{i} \tilde{\psi}+\left\langle b, \partial_{i} b\right\rangle\right)\left(\partial_{j} \tilde{\psi}+\left\langle b, \partial_{j} b\right\rangle\right)
\end{aligned}
$$

and of the following ones:

$$
\begin{aligned}
& \partial_{i}\left[\sqrt{\gamma} \gamma^{i j} e^{-4 U}\left(\partial_{j} \tilde{\psi}+\left\langle b, \partial_{j} b\right\rangle\right)\right]=0, \\
& \partial_{i}\left[\sqrt{\gamma} \gamma^{i j} e^{-2 U} M_{\alpha \beta} \partial_{j} b^{\beta}\right]=\partial_{i}\left[\sqrt{\gamma} \gamma^{i j} e^{-4 U} \Omega_{\alpha \beta} b^{\beta}\left(\partial_{j} \tilde{\psi}+\left\langle b, \partial_{j} b\right\rangle\right)\right], \\
& \frac{1}{\sqrt{\gamma}} \partial_{i}\left[\sqrt{\gamma} \gamma^{i j} \partial_{j} U\right]=-\frac{1}{2} e^{-2 U} \gamma^{i j} \partial_{i} b^{\alpha} M_{\alpha \beta} \partial_{j} b^{\beta} \\
& -\frac{1}{2} e^{-4 U} \gamma^{i j}\left(\partial_{i} \tilde{\psi}+\left\langle b, \partial_{i} b\right\rangle\right)\left(\partial_{j} \tilde{\psi}+\left\langle b, \partial_{j} b\right\rangle\right), \\
& \frac{1}{\sqrt{\gamma}} \partial_{i}\left[\sqrt{\gamma} \gamma^{i j} G_{a \bar{a}} \partial_{j} \bar{z}^{\bar{a}}\right]=\frac{\partial G_{b \bar{b}}}{\partial z^{a}} \gamma^{i j} \partial_{i} z^{b} \partial_{j} \bar{z}^{\bar{b}}+\frac{1}{2} e^{-2 U} \gamma^{i j} \partial_{i} b^{\alpha} \frac{\partial M_{\alpha \beta}}{\partial z^{a}} \partial_{j} b^{\beta} .
\end{aligned}
$$

Within the class of the stationary metrics (2.2), we further select those with $D=3$ Euclidean metric $\gamma_{i j}=\delta_{i j}$ :

$$
d s^{2}=e^{2 U}\left(d t+\omega_{i} d x^{i}\right)^{2}-e^{-2 U} d x^{i} d x^{i} .
$$

Thus, we will henceforth restrict to consider $D=4$ under-rotating BHs, with flat $D=3$ base space. When replacing (2.2) with (2.11), Einstein equations (2.9) become a first order constraint:

$G_{a \bar{a}} \partial_{(i} z^{a} \partial_{j)} \bar{z}^{\bar{a}}+\partial_{i} U \partial_{j} U+\frac{1}{2} e^{-2 U} \partial_{i} b^{\top} M \partial_{j} b+\frac{1}{4} e^{-4 U}\left(\partial_{i} \tilde{\psi}+\left\langle b, \partial_{i} b\right\rangle\right)\left(\partial_{j} \tilde{\psi}+\left\langle b, \partial_{j} b\right\rangle\right)=0$,

and the equations of motion (2.10) get slightly simplified:

$$
\begin{aligned}
\partial_{i}\left[e^{-4 U}\left(\partial_{i} \tilde{\psi}+\left\langle b, \partial_{i} b\right\rangle\right)\right] & =0 \\
\partial_{i}\left[e^{-2 U} M_{\alpha \beta} \partial_{i} b^{\beta}\right] & =\partial_{i}\left[e^{-4 U} \Omega_{\alpha \beta} b^{\beta}\left(\partial_{i} \tilde{\psi}+\left\langle b, \partial_{i} b\right\rangle\right)\right] \\
\partial_{i} \partial_{i} U & =-\frac{1}{2} e^{-2 U} \partial_{i} b^{\top} M \partial_{i} b-\frac{1}{2} e^{-4 U}\left(\partial_{i} \tilde{\psi}+\left\langle b, \partial_{i} b\right\rangle\right)^{2}, \\
\partial_{i}\left[G_{a \bar{a}} \partial_{i} \bar{z}^{\bar{a}}\right] & =\frac{\partial G_{b \bar{b}}}{\partial z^{a}} \partial_{i} z^{b} \partial_{i} \bar{z}^{\bar{b}}+\frac{1}{2} e^{-2 U} \partial_{i} b^{\top} \frac{\partial M}{\partial z^{a}} \partial_{i} b .
\end{aligned}
$$

As a notation, let us introduce a $D=3$ vector $\chi_{i}$, which, by virtue of $(2.13 \mathrm{a})$, is divergenceless:

$$
\chi_{i} \equiv e^{-4 U}\left(\partial_{i} \tilde{\psi}+\left\langle b, \partial_{i} b\right\rangle\right), \quad \partial_{i} \chi_{i}=0 .
$$

Maxwell equations (2.13b) can immediately be integrated, yielding:

$$
\partial_{i} b^{\alpha}=e^{2 U} M^{\alpha \beta} \Omega_{\beta \gamma} \hat{H}_{i}^{\gamma}, \quad \hat{H}_{i}^{\alpha} \equiv H_{i}^{\alpha}+b^{\alpha} \chi_{i}, \quad \partial_{i} H_{i}^{\alpha}=0,
$$


where another $D=3$ divergenceless vector $H_{i}^{\alpha}$ has been defined. The electromagnetic two-form field strength $f_{i j}^{\alpha}$ and the two-form $W_{i j}$ corresponding to rotation are then just Hodge duals of the vectors $\chi_{i}$ and $\hat{H}_{i}^{\alpha}$ defined in (2.14)-(2.15):

$$
W_{i j}=-\varepsilon_{i j k} \chi_{k}, \quad f_{i j}^{\alpha}=-\varepsilon_{i j k} \hat{H}_{k}^{\alpha}-b^{\alpha} W_{i j}=-\varepsilon_{i j k} H_{k}^{\alpha} .
$$

This allows one to rewrite the Einstein equations (2.12) as well as the rest of the equations of motion (2.13) as follows:

$$
\begin{gathered}
G_{a \bar{a}} \partial_{(i} z^{a} \partial_{j)} \bar{z}^{\bar{a}}+\partial_{i} U \partial_{j} U-\hat{V}_{i j} e^{2 U}+\frac{1}{4} e^{4 U} \chi_{i} \chi_{j}=0, \\
\partial_{i} \partial_{i} U=e^{2 U} \hat{V}_{i i}-\frac{1}{2} e^{4 U} \chi_{i}^{2} \\
\partial_{i}\left(G_{a \bar{a}} \partial_{i} \bar{z}^{\bar{a}}\right)=\partial_{a} G_{b \bar{b}} \partial_{i} z^{b} \partial_{i} \bar{z}^{\bar{b}}+e^{2 U} \partial_{a} \hat{V}_{i i} .
\end{gathered}
$$

In (2.16)-(2.17) the $D=3$ Cartesian tensor

$$
\hat{V}_{i j} \equiv-\frac{1}{2} \hat{H}_{i}^{\top} M \hat{H}_{j}
$$

has been introduced; it will be referred to as black hole potential. In fact, it generalizes, for the whole class of metrics (2.11), the well known BH potential $V_{B H}$.

This latter was introduced in [30] for the case of a static and spherically symmetric BH. In our approach, this case corresponds to a vanishing $\chi_{i}$ and to the choice of the divergenceless vector $H_{i}^{\alpha}$ to be the gradient of an harmonic function:

$$
\chi_{i}=0, \quad H_{i}^{\alpha}=\partial_{i} H^{\alpha} .
$$

In the case of spherical symmetry, $H^{\alpha}$ has a single pole where the black hole horizon resides (i.e. in the origin):

$$
H^{\alpha}(x, y, z)=h^{\alpha}+P^{\alpha} \tau, \quad h^{\alpha}=\text { const }, \quad \tau=\frac{1}{\sqrt{x^{2}+y^{2}+z^{2}}} .
$$

The constants $P^{\alpha}$ fit into the symplectic vector of electromagnetic charges $\left(p^{\Lambda}, q_{\Lambda}\right)$ of the $\mathrm{BH}$ itself. Within these assumptions, the tensor $\hat{V}_{i j}$ relates to $V_{B H}$ as follows:

$$
\hat{V}_{i j}=\partial_{i} \tau \partial_{j} \tau V_{B H}, \quad V_{B H}=-\frac{1}{2} P^{\top} M P .
$$

Moreover, Maxwell eqs. (2.15) decouple completely from (2.16) and (2.17), which get exactly the form presented in [30]. Therein, it was also shown that in the case in which the action (2.1) describes the bosonic sector of $N=2, D=4$ Maxwell-Einstein supergravity, the BH potential $V_{B H}$ acquires a nice geometrical interpretation in terms of special Kähler geometry (see e.g. [31] for a review and a list of refs.):

$$
V_{B H}=Z \bar{Z}+G^{a \bar{a}} D_{a} Z \bar{D}_{\bar{a}} \bar{Z}
$$


where $Z$ is the $N=2$ central charge function and $D_{a} Z$ (matter charges) denotes its Kähler-covariant derivative:

$$
Z=\langle P, V\rangle, \quad D_{a} Z=\left\langle P, D_{a} V\right\rangle,
$$

defined in terms of the symplectic sections $V^{\alpha}$ of the flat symplectic bundle of special geometry $[32,33]$.

Interestingly, the Cartesian $D=3$ tensor $\mathrm{BH}$ potential $\hat{V}_{i j}(2.18)$ can be given a geometrical interpretation in the spirit of eq. (2.22). Indeed, if one constructs a $D=3$ Cartesian generalization of the central charge and of its covariant derivatives as

$$
\hat{Z}_{i} \equiv\left\langle\hat{H}_{i}, V\right\rangle, \quad \hat{Z}_{a i} \equiv\left\langle\hat{H}_{i}, D_{a} V\right\rangle,
$$

then it can be computed that

$$
\hat{V}_{i j}=\hat{Z}_{(i} \hat{\bar{Z}}_{j)}+G^{a \bar{a}} \hat{Z}_{a(i} \hat{\bar{Z}}_{\bar{a} j)},
$$

which can be regarded as the generalization of (2.22) to generic, not necessarily axisymmetric, and thus possibly multi-centered, stationary solutions (at least those with flat three-dimensional spatial slices, cfr. (2.11)).

For later convenience, let us also here define the non-rotating limit $\left(\chi_{i}=0\right)$ of $\hat{Z}_{i}$ and $\hat{Z}_{a i}$ defined in $(2.24)$ :

$$
Z_{i}=\left\langle H_{i}, V\right\rangle, \quad Z_{a i}=\left\langle H_{i}, D_{a} V\right\rangle .
$$

As it will become evident from the subsequent treatment, both Cartesian $D=3$ generalizations (2.24) and (2.26) of the $N=2$ central charge $Z$ and of its covariant derivatives $D_{a} Z$ will play an important role in the construction of first order equations of motion for multi-centered BHs.

\section{First order equation of motion}

The first order equations of motion arise from the following Ansatz:

$$
\partial_{i} U=e^{U} W_{i}, \quad \partial_{i} z^{a}=e^{U} \Pi_{i}^{a}, \quad \chi_{i}=2 e^{-U} \ell_{i},
$$

where $W_{i}, \Pi_{i}^{a}$ and $\ell_{i}$ are functions of the scalar fields and, eventually, of some auxiliary ones. The choice of the Ansatz (3.1) is motivated by the fact that for single- and multicentered BPS, as well for single-centered non-BPS BHs, the first order equations are of the form (3.1).

In the following treatment, we will conveniently consider special Kähler geometry tensors whose indices are "flattened" as usual:

$$
V_{\underline{a}} \equiv E_{\underline{a}^{a}} D_{a} V, \quad \hat{Z}_{\underline{a}} i \equiv \underline{E}^{a} \hat{Z}_{a i} \quad \text { etc. }
$$

by using the Vielbein of the scalar manifold (for further details, see appendix A). By means of (3.2), the first order equations (3.1) can be recast as follows:

$$
\partial_{i} U=e^{U} W_{i}, \quad \partial_{i} z^{a}=e^{U} \Pi_{i}^{\underline{a}} E_{\underline{a}}^{a}, \quad \chi_{i}=2 e^{-U} \ell_{i} .
$$


Due to the Einstein equations (2.16), the functions entering the right hand sides of eqs. (3.3) must satisfy the algebraic constraint

$$
\hat{V}_{i j}=W_{i} W_{j}+\delta_{\underline{a}} \underline{\bar{a}} \Pi \frac{a}{(i} \bar{\Pi}_{j}^{\bar{a}}+\ell_{i} \ell_{j}
$$

expressing the relation between $\hat{V}_{i j}$ and the $D=3$ Cartesian fake superpotential $W_{i}$, and generalizing the relation [18]

$$
V_{B H}=W^{2}+4 G^{a \bar{a}} \partial_{a} W \bar{\partial} \bar{a} W
$$

to which it reduces in the limit ${ }^{3}(2.19)-(2.21)$.

For later convenience, let us define the complex $D=3$ Cartesian vector

$$
\mathcal{W}_{i} \equiv W_{i}+i \ell_{i}
$$

in terms of which the algebraic constraint (3.4) can be recast $\operatorname{as}^{4}$ (cfr. (2.25))

$$
\hat{V}_{i j}=\mathcal{W}_{(i} \overline{\mathcal{W}}_{j)}+\delta_{\underline{a}} \underline{\bar{a}} \Pi \frac{a}{(i} \bar{\Pi}_{j)}^{\bar{a}}
$$

We anticipate that the complex vector $\mathcal{W}_{i}$ will play an important role in integrating Maxwell eqs. (2.15).

From now on, in order to avoid overloading the formulae, we omit underlining the flat indices.

\subsection{Construction of flows}

We are now going to determine the first order flow-governing functions $W_{i}, \ell_{i}$ (or $\mathcal{W}_{i}$ ) and $\Pi_{i}^{a}$.

A crucial step in constructing such flow-governing functions is to use their expression in the BPS case, and then perform a suitable flipping of some (linear combinations of) electromagnetic charges.

\subsubsection{BPS}

As it is known [1, 18], in the BPS class [16], at spatial infinity (i.e., where one may think of a restoration of the spherical symmetry), the first order superpotential $W$ is just a combination of the ADM mass $M$ and the NUT charge $N$, while the functions $\Pi^{a}$ are the scalar charges ${ }^{5}$

$$
W=\operatorname{Re}(M+i N), \quad \Pi^{a}=\pi^{a} .
$$

In turn, at spatial infinity it holds

$$
M+i N=Z, \quad \pi^{a}=\bar{Z}_{\bar{a}},
$$

\footnotetext{
${ }^{3}$ Within the same limit, the first order constraint (2.12) reduces to the Hamiltonian constraint given by eq. (11) (with $c=0$ ) of [30].

${ }^{4}$ For simplicity's sake, starting from below (3.7) we will refrain from underlining the scalar flat indices. In presence of curved indices, we hope the distinction will be clear from the context.

${ }^{5}$ Here, we omit the spatial index $i$ because in the case under consideration the spherical symmetry reduces the number of independent components of a Cartesian vector to one.
} 
and thus (3.8) can be rewritten as:

$$
W=\operatorname{Re} Z, \quad \Pi^{a}=\bar{Z}_{\bar{a}} .
$$

In order to proceed further, we will now specialize our treatment to the $N=2, D=4$ stu model [27, 28], which can also be regarded as a common sector of $D=4$ supergravity theories with rank-3 symmetric scalar manifolds.

Within this model, in order to restore manifest duality covariance in (3.10), one has to consider the proper action of the compact symmetry $[\mathrm{U}(1)]^{4}=H_{4} \times \mathrm{U}(1)$, where $H_{4}$ is the stabilizer of the completely factorized $D=4$ scalar manifold $[\mathrm{SL}(2, \mathbb{R}) / \mathrm{U}(1)]^{3}$, or equivalently the maximal compact subgroup $(\mathrm{mcs})$ of the $D=4$ generalized electricmagnetic $\left(U\right.$-)duality group $G_{4}=[\mathrm{SL}(2, \mathbb{R})]^{3}$, while the commuting $\mathrm{U}(1)$ is the $m c s$ of the $\mathrm{SL}(2, \mathbb{R})$ Ehlers symmetry determined by the reduction to tree dimensions. As discussed in $[1]$, under the resulting $[\mathrm{U}(1)]^{4}$ symmetry, the central charge and its derivatives transform as follows 6

$$
Z \rightarrow e^{-\frac{i}{2}\left(\alpha_{0}-\sum_{a} \alpha_{a}\right)} Z, \quad Z_{a} \rightarrow e^{-\frac{i}{2}\left(\alpha_{0}-\alpha_{a}+\sum_{b \neq a} \alpha_{b}\right)} Z_{a},
$$

while the ADM mass $M$, NUT charge $N$ and scalar charges $\pi^{a}$ transform as

$$
M+i N \rightarrow e^{\frac{i}{2}\left(\alpha_{0}+\sum_{a} \alpha_{a}\right)}(M+i N), \quad \pi^{a} \rightarrow e^{-\frac{i}{2}\left(\alpha_{0}+\alpha_{a}-\sum_{b \neq a} \alpha_{b}\right)} \pi^{a} .
$$

Therefore, in order to restore manifest covariance in eq. (3.10), one applies transformations (3.11) on the left and right hand sides of eq. (3.9):

$$
e^{\frac{i}{2}\left(\alpha_{0}+\sum_{a} \alpha_{a}\right)}(M+i N)=e^{-\frac{i}{2}\left(\alpha_{0}-\sum_{a} \alpha_{a}\right)} Z, \quad e^{-\frac{i}{2}\left(\alpha_{0}+\alpha_{a}-\sum_{b \neq a} \alpha_{b}\right)} \pi^{a}=e^{\frac{i}{2}\left(\alpha_{0}-\alpha_{a}+\sum_{b \neq a} \alpha_{b}\right)} \bar{Z}_{\bar{a}} ;
$$

by some trivial algebra, and taking into account eq. (3.8), one achieves the following result, depending only on the phase $\alpha_{0}$ :

$$
W=\operatorname{Re}\left(e^{-i \alpha_{0}} Z\right), \quad \Pi^{a}=e^{i \alpha_{0}} \bar{Z}_{\bar{a}} .
$$

Although eqs. (3.14) were obtained in [1] at spatial infinity only, we will postulate that they are valid not only at the spatial infinity, but all along the corresponding whole scalar flow (with generally broken spherical symmetry), and we will study the consequences of this approach in the next sections. Our approach then justifies the following "Cartesian generalization" of (3.14):

$$
W_{i}=\operatorname{Re}\left(e^{-i \alpha_{0}} \hat{Z}_{i}\right), \quad \Pi_{i}^{a}=e^{i \alpha_{0}} \hat{\bar{Z}}_{\bar{a} i}
$$

with $\hat{Z}_{i}$ and $\hat{Z}_{a i}$ defined in (2.24). Consequently, the function $\ell_{i}$, and hence $\chi_{i}$, is easily deduced from the Einstein constraint (3.7):

$$
\ell_{i}=\operatorname{Im}\left(e^{-i \alpha_{0}} \hat{Z}_{i}\right) \quad \Rightarrow \quad \chi_{i}=2 e^{-U} \operatorname{Im}\left(e^{-i \alpha_{0}} \hat{Z}_{i}\right) .
$$

\footnotetext{
${ }^{6}$ The phases $\alpha_{0}$ and $\alpha_{a}(a=1,2,3)$ are not directly related to the Ehlers $\mathrm{U}(1)$ and to the three U(1)'s in $H_{4}$, but rather they are a linear combinations of the corresponding phases thereof; for further detail, see $[1]$.
} 
Therefore, one realizes that the complex vector $M+i N$ (composed by the ADM mass $M$ and the NUT charge $N$ ) can be "prolonged" all along the scalar flow by the Cartesian $D=3$ vector $\mathcal{W}_{i}(3.6)$, which in this case reads

$$
\mathcal{W}_{i}=W_{i}+i \ell_{i}=e^{-i \alpha_{0}} \hat{Z}_{i}
$$

The phase $\alpha_{0}$ becomes a dynamical field and the consistency of the first order equations require that it satisfy the following equation

$$
\partial_{i} \alpha_{0}=e^{U} \operatorname{Im}\left(e^{-i \alpha_{0}} \hat{Z}_{i}\right)-\operatorname{Im}\left(\partial_{a} K \partial_{i} z^{a}\right) .
$$

It is here worth stressing that eqs. (3.16) resemble the ones obtained in [16], but there is an important difference: they are indeed expressed in terms of $\hat{Z}_{i}$, instead of $Z_{i}$. The very definition (2.24) of the $D=3$ Cartesian $N=2$ central charge $\hat{Z}_{i}$ involves terms proportional to the vector $\chi_{i}$, thus eq. (3.16) can actually be solved for $\chi_{i}$ itself, achieving the following expression:

$$
\chi_{i}=\frac{2 e^{-U} \operatorname{Im}\left[e^{-i \alpha_{0}} Z_{i}\right]}{1-2 e^{-U} \operatorname{Im}\left[e^{-i \alpha_{0}}\langle b, V\rangle\right]} .
$$

Then, the integration of Maxwell eqs. (2.15) exactly implies the result of [16]. In order to achieve this, one is hinted by the fact that expression (3.15) can be recast in the following form:

$$
W_{i}=\operatorname{Re}\left\langle\hat{H}_{i}, e^{-i \alpha_{0}} V\right\rangle
$$

and one can thus check that

$$
b^{\alpha}=2 e^{U} \operatorname{Re}\left(e^{-i \alpha_{0}} V^{\alpha}\right)
$$

satisfies the Maxwell equations (2.15), thus yielding the following flow-defining functions:

$$
W_{i}=\operatorname{Re}\left(e^{-i \alpha_{0}} Z_{i}\right), \quad \Pi_{i}^{a}=e^{i \alpha_{0}} \bar{Z}_{\bar{a} i}, \quad \chi_{i}=-2 e^{-U} \operatorname{Im}\left(e^{-i \alpha_{0}} Z_{i}\right),
$$

which govern the first-order formulation of scalar flows of the BPS class of multi-centered BHs [16].

\subsubsection{Almost BPS}

In order to construct a first order formalism for non-BPS flows, we will exploit suitable charge flippings at spatial infinity, and then consistently extend them all along the flow. The relevant flippings of charges have been derived in [1] by exploiting an analysis of the relevant nilpotent orbits of $\mathrm{SO}(4,4)$, which is the $D=3$ duality group of the stu model.

We start and consider the central charge $Z$ and its flat derivatives $Z_{a}$ at spatial infinity. Without loss of generality (by $H_{4}=\mathrm{U}(1)^{3}$ duality), the asymptotical values of the scalar fields $z^{a}$ can be set to

$$
z^{a}=-i
$$

Therefore, at spatial infinity the central charge along with its flat Kähler-covariant derivatives respectively read

$$
Z=\frac{1}{2 \sqrt{2}}\left[q_{0}+i p^{0}-\sum_{a}\left(p^{a}-i q_{a}\right)\right], \quad Z_{a}=\frac{1}{2 \sqrt{2}}\left[q_{0}-i p^{0}+\sum_{b \neq a}\left(p^{b}+i q_{b}\right)-p^{a}+i q_{a}\right] .
$$


Instead of dealing with the charges $\left(p^{0}, p^{a}, q_{0}, q_{a}\right)$, let us define a basis $\left(D^{0}, D^{a}, D_{0}, D_{a}\right)$ whose interpretation in terms of $D$-brane charges is given below [1]:

$$
\begin{array}{ll}
\mathrm{D} 6: D^{0} \equiv \frac{1}{2}\left[p^{0}+\sum_{a} q_{a}\right], & \mathrm{D} 4: D^{a} \equiv p^{a}, \\
\mathrm{D} 2: D_{a} \equiv \frac{1}{2}\left[p^{0}+q_{a}-\sum_{b \neq a} q_{b}\right], & \text { D0 }: D_{0} \equiv q_{0} ;
\end{array}
$$

this is a more convenient basis on which one can act with the charge flipping. In terms of $D$-charges (3.23), (3.22) can be rewritten as

$$
\begin{aligned}
& Z=\frac{1}{2 \sqrt{2}}\left[D_{0}-i D^{0}+\sum_{a}\left(D^{a}+i D_{a}\right)\right], \\
& Z_{a}=\frac{1}{2 \sqrt{2}}\left[D_{0}-i D^{0}-\sum_{b \neq a}\left(D^{b}+i D_{b}\right)+D^{a}+i D_{a}\right],
\end{aligned}
$$

or equivalently, inverting in terms of the $D$-charges:

$$
\begin{array}{ll}
D_{0}=\frac{1}{\sqrt{2}} \operatorname{Re}\left[Z+\sum_{a} Z_{a}\right], & D_{a}=\frac{1}{\sqrt{2}} \operatorname{Im}\left[Z+Z_{a}-\sum_{b \neq a} Z_{b}\right], \\
D^{0}=-\frac{1}{\sqrt{2}} \operatorname{Im}\left[Z+\sum_{a} Z_{a}\right], & D^{a}=\frac{1}{\sqrt{2}} \operatorname{Re}\left[Z+Z_{a}-\sum_{b \neq a} Z_{b}\right] .
\end{array}
$$

Now, consistent with the analysis of [1], let us flip the sign of the brane charge $D_{0}(3.23)$ in (3.24), obtaining

$$
\begin{aligned}
& \left.\widetilde{Z} \equiv Z\right|_{D_{0} \rightarrow-D_{0}}=\frac{1}{2 \sqrt{2}}\left[-D_{0}-i D^{0}+\sum_{a}\left(D^{a}+i D_{a}\right)\right] \\
& \left.\widetilde{Z}_{a} \equiv Z_{a}\right|_{D_{0} \rightarrow-D_{0}}=\frac{1}{2 \sqrt{2}}\left[-D_{0}-i D^{0}-\sum_{b \neq a}\left(D^{b}+i D_{b}\right)+D^{a}+i D_{a}\right]
\end{aligned}
$$

by plugging the expressions (3.25) into (3.26), one achieves the following result:

$$
\widetilde{Z}=\frac{1}{4}\left[3 Z-\bar{Z}-2 \operatorname{Re} \sum_{a} Z_{a}\right], \quad \widetilde{Z}_{a}=-\frac{1}{2}\left[\operatorname{Re} Z+\operatorname{Re} \sum_{b} Z_{b}-2 Z_{a}\right],
$$

which can then be plugged into the right hand side of eqs. (3.9). By restoring manifest $(\mathrm{U}(1))^{4}$ covariance in the way described above, and extending these relations along the whole flow and not only at spatial infinity, one obtains the following expressions, explicitly depending on all four phases $\alpha_{0}$ and $\alpha_{a}$ 's:

$$
\begin{aligned}
& W_{i}=\frac{1}{4} \operatorname{Re}\left[e^{-i \alpha_{0}}\left(3-e^{i\left(\alpha_{0}+\sum_{a} \alpha_{a}\right)}\right) \hat{Z}_{i}-\left(1+e^{-i\left(\alpha_{0}+\sum_{a} \alpha_{a}\right)}\right) \sum_{a} e^{i \alpha_{a}} \hat{Z}_{a i}\right] \\
& \Pi_{i}^{a}=e^{i \alpha_{0}} \hat{\bar{Z}}_{a i}-\frac{1}{2} e^{\frac{i}{2}\left(\alpha_{0}+\alpha_{a}-\sum_{b \neq a} \alpha_{b}\right)} \operatorname{Re}\left(e^{-\frac{i}{2}\left(\alpha_{0}-\sum_{b} \alpha_{b}\right)} \hat{Z}_{i}+\sum_{b} e^{-\frac{i}{2}\left(\alpha_{0}-\alpha_{b}+\sum_{c \neq b} \alpha_{c}\right)} \hat{Z}_{b i}\right) .
\end{aligned}
$$


This case corresponds to the so-called almost BPS class [1,3], whose first order formulation will be studied in section 5 .

\subsubsection{Composite non-BPS}

Another possibility is to flip not only $D_{0}$, but also $D_{a}$ brane charges (3.23) as well, namely:

$$
D_{0} \rightarrow-D_{0}, \quad D_{a} \rightarrow-D_{a} .
$$

By applying the very same procedure described above for the sign flip (3.29), one finally gets the following expressions, explicitly depending on onlythree phases $\alpha_{a}$ 's:

$$
\begin{aligned}
& W_{i}=\frac{1}{2} \operatorname{Re}\left(e^{i \sum_{a} \alpha_{a}} \hat{Z}_{i}-\sum_{a} e^{i \alpha_{a}} \hat{Z}_{a i}\right) \\
& \Pi_{i}^{a}=-\frac{1}{2}\left[e^{i \alpha_{a}} \hat{Z}_{i}-e^{i\left(\alpha_{a}-\sum_{b \neq a} \alpha_{b}\right)} \hat{Z}_{a i}+\sum_{b \neq a} e^{-i \alpha_{b}} \hat{Z}_{b i}\right] .
\end{aligned}
$$

This case corresponds to the so-called composite non-BPS class [1], whose first order formulation will be studied in section 4 .

\section{Composite non-BPS class}

We will start analyzing the composite non-BPS [1] class (3.30), its structure being relatively simpler than the one of the almost-BPS class.

We shall generalize the results of [25], matching the treatment given in [12] in the case of the stu model (cfr. section 4.1 therein).

In the previous section, we constructed the functions $W_{i}$ and $\Pi_{i}^{a}(3.30)$, governing the first order flow eqs. (3.1). From Einstein eqs. (2.16), one can deduce the following expression for the remaining flow-governing function, namely the $D=3$ Cartesian rotation vector $\ell_{i}$ :

$$
\ell_{i}=-\frac{1}{2} \operatorname{Im}\left[e^{i \sum_{a} \alpha_{a}} \hat{Z}_{i}-\sum_{a} e^{i \alpha_{a}} \hat{Z}_{a i}\right] .
$$

As it can be realized by looking at previous equations, the various $\alpha$-phases play an important role in the first order formalism for the multi-centered scalar flows. Nevertheless, their own dynamics has not been concerned so far. In the case This can be uniquely determined if one imposes that after differentiation of the first order equations (3.3), (3.30) and (4.1), the second order ones (2.17) are obtained. This requirement yields the following three partial differential eqs. for the three phases $\alpha_{a}$ 's $(a=1,2,3)$ in the composite nonBPS multi-centered class: ${ }^{7}$

$$
\begin{aligned}
\partial_{i} \alpha_{a}=\frac{1}{2} e^{U} \operatorname{Im} & {\left[\left(e^{i \sum_{b} \alpha_{b}}+e^{i \alpha_{a}}-\sum_{b \neq a} e^{i \alpha_{b}}\right) \hat{Z}_{i}+2 e^{i \alpha_{a}} \hat{Z}_{a i}-2 e^{-i \alpha_{a}} \sum_{b \neq a} \hat{Z}_{b i}\right.} \\
& \left.+e^{-i \alpha_{a}}\left(e^{-i \sum_{b} \alpha_{b}}-e^{i \alpha_{a}}+\sum_{b \neq a} e^{-i \alpha_{b}}\right)\left(-e^{i \alpha_{a}} \hat{Z}_{a i}+\sum_{b \neq a} e^{i \alpha_{b}} \hat{Z}_{b i}\right)\right] .
\end{aligned}
$$

\footnotetext{
${ }^{7}$ It is worth noticing that both sets (4.2) and (5.2) of partial differential eqs. for the phases (respectively in the composite non-BPS e almost BPS classes) exhibit a manifest stu triality symmetry [27, 28].
} 
Thus, one can conclude that eqs. (3.3), (3.30), (4.1), and (4.2) - together with Maxwell eqs. (2.15) - constitute the first order formalism for composite non-BPS multi-centered $B H$ solutions in $N=2, D=4$ stu model.

Let us now integrate further Maxwell eqs. (2.15) by exploiting the hint described in section 3.1.1 for the BPS flow. Namely, one can easily notice that for the composite nonBPS class the complex $D=3$ Cartesian vector $\mathcal{W}_{i}(3.6)$ can be rewritten as

$$
\mathcal{W}_{i}=\left\langle\hat{H}_{i}, T\right\rangle,
$$

where the complex symplectic vector

$$
T^{\alpha} \equiv \frac{1}{2}\left(e^{-i \sum_{a} \alpha_{a}} \bar{V}^{\alpha}-\sum_{a} e^{-i \alpha_{a}} \bar{V}_{\bar{a}}^{\alpha}\right)
$$

was defined. After a little algebra, one also finds that electromagnetic potential $b(2.6)$ enjoys an expression similar to the one holding for the BPS class (3.19), namely:

$$
b^{\alpha}=2 e^{U} \operatorname{Re}\left[T^{\alpha}\left(1-i B e^{2 U}\right)\right],
$$

for some function $B$, which can be considered as the $D=5$ rotational contribution to the electromagnetic potential.

As it occurred in the BPS multi-centered class treated in section 3.1.1, also in the composite non-BPS class eq. (4.1) contains $\chi_{i}$ in both sides. Remarkably, in this class the dependence on $\chi_{i}$ drops out when replacing (4.5) into (4.1); as a consequence, eq. (4.1) turns into the following algebraic constraint:

$$
\operatorname{Im}\left(e^{i \sum_{a} \alpha_{a}} Z_{i}-\sum_{a} e^{i \alpha_{a}} Z_{a i}\right)=0
$$

and the $D=3$ Cartesian rotation vector $\chi_{i}$ can be computed to read

$$
\chi_{i}=\partial_{i} B+B e^{U} \operatorname{Re}\left(3 e^{i \sum_{a} \alpha_{a}} Z_{i}-\sum_{a} e^{i \alpha_{a}} Z_{a i}\right)-2 e^{-U} \operatorname{Im}\left(e^{i \sum_{a} \alpha_{a}} Z_{i}\right),
$$

where the function $B$ occurred for the first time in (4.5), and it should be such that $\chi_{i}$ is divergenceless (cfr. (2.14)).

The counting of the two "flat" directions along the (non-BPS, "large") flows in the stu model $[23,35]$ is retrieved by considering that in this class the tree partial differential eqs. (4.2) for the tree phases $\alpha_{a}$ 's are supplemented by the algebraic constraint (4.6) (see also the treatment of next subsection).

\subsection{Single-centered solutions}

Consistent with [1] (see also e.g. the two-centered analysis in [34]), we will now show that the well known case of a single-centered non-rotating non-BPS BH can be obtained by performing a suitable limit in the multi-centered class under consideration. 
We consider the single-centered limit (2.19)-(2.20), in which the divergenceless vector $H_{i}^{\alpha}$ introduced in (2.15) is realized as a derivative of an harmonic function $H^{\alpha}$ defined in $(2.20)$ :

$$
H_{i}^{\alpha}=\partial_{i} H^{\alpha}, \quad \partial_{i} \partial_{i} H^{\alpha}=0 .
$$

Before proceeding further, it is worth clarifying here the meaning of the constraint (4.6). With such a purpose, let us introduce the three real values

$$
\lambda^{a} \equiv \frac{\operatorname{Im}\left(e^{i \beta_{a}} z^{a}\right)}{\operatorname{Im}\left(e^{i \beta_{a}}\right)}, \quad \beta_{a} \equiv \frac{1}{2} \sum_{b \neq a} \alpha_{b}
$$

and construct an stu-like symplectic section

$$
V_{\lambda}^{\alpha} \equiv\left(1, \lambda^{1}, \lambda^{2}, \lambda^{3},-\lambda^{1} \lambda^{2} \lambda^{3}, \lambda^{2} \lambda^{3}, \lambda^{1} \lambda^{3}, \lambda^{1} \lambda^{2}\right)^{T}
$$

This allows the following rather elegant rewriting of the constraint (4.6):

$$
\left\langle P, V_{\lambda}\right\rangle=0
$$

Note that the vector $V_{\lambda}$, which can consistently be defined only in the composite non-BPS class, coincides with the doubly critical vector $R$ introduced in [12], and firstly discussed in the second ref. of $[14,15]$. Moreover, the three $\lambda^{a}$ 's satisfy the single constraint (4.11), and therefore only two of them are independent; this reflects the existence of two "flat" directions along the non-rotating single-centered stu non-BPS flow [23, 35]. A noteworthy feature of $\lambda^{a}$ 's is that they are in fact integrals of motion in $D=3$ flat space; indeed, from the above eqs. one can check that

$$
\partial_{i} \lambda^{a}=0
$$

By inverting the definitions (4.9), one can thus express the three phases $\alpha_{a}$ 's in terms of the scalar fields $z^{a}$ and of the integrals of motion $\lambda^{a}$ 's themselves:

$$
e^{i \sum_{b \neq a} \alpha_{b}}=\frac{\lambda^{a}-\bar{z}^{\bar{a}}}{\lambda^{a}-z^{a}}
$$

In the case of spherical symmetry, each of the flow-defining $D=3$ Cartesian vectors $W_{i}$ and $\Pi_{i}^{a}$ can actually be reduced to only one independent function:

$$
W_{i}=\partial_{i} \tau W, \quad \Pi_{i}^{a}=\partial_{i} \tau \Pi^{a},
$$

where $\tau$ has been defined in (2.20); therefore, the spatial vector index $i$ can formally be neglected.

By plugging the phases (4.13) into the eqs. (3.30), one retrieves the known expression for the non-BPS fake superpotential $W_{n B P S}$ for a non-rotating single-centered non-BPS BH in stu model (with non-vanishing $Z$ at the horizon) [23]; in order to achieve a complete agreement in notations, one should parametrize the integrals of motion as

$$
\lambda^{a} \equiv \frac{e^{\alpha_{a}} \nu \xi_{a}+\rho_{a}}{e^{\alpha_{a}} \nu-1}
$$


where the $\alpha_{a}$ 's are some constant (not to be confused with the phases $\alpha_{a}$ 's) satisfying $\sum_{a} \alpha_{a}=0$.

It should be here remarked that in the non-rotating $\left(\chi_{i}=0\right)$ single-centered non-BPS BH solution supported by the Kaluza-Klein $\left(p^{0}, q_{0}\right)$-configuration $[23,36]$

$$
\begin{aligned}
e^{-4 U} & =H_{0} H_{1} H_{2} H_{3}-B^{2}, & z^{a} & =\frac{A_{a}^{-}-2 i e^{-2 U}}{A_{a}^{+}-2 B} ; \\
B & =\text { const }, & A_{a}^{ \pm} & =\frac{H_{1} H_{2} H_{3}}{H_{a}} \pm H_{0} H_{a},
\end{aligned}
$$

the function $\chi_{i}$, defined by eq. (4.7), vanishes. On the other hand, if the function $B$ is not a constant, then it turns out to read

$$
B=b+J \frac{\cos \theta}{r^{2}}
$$

where $J$ is an angular momentum; this corresponds to the Rasheed-Larsen rotating $\left(\chi_{i} \neq 0\right)$ single-centered non-BPS BH $[37,38]$.

\subsection{Multi-centered solutions}

\subsubsection{Electric configuration}

In [1], it was found that for the composite non-BPS class there exists a particular solution

$$
e^{-4 U}=4 V_{0} H_{1} H_{2} H_{3}-B^{2}, \quad z^{a}=\frac{1}{2} \frac{2 K_{a} \frac{H_{1} H_{2} H_{3}}{H_{a}}-B-i e^{-2 U}}{K_{a}^{2} \frac{H_{1} H_{2} H_{3}}{H_{a}}+H_{a} V_{0}-B K_{a}},
$$

defined in terms of functions $V_{0}, H_{a}$ and $K_{a}$ satisfying the following equations:

$$
\begin{aligned}
\partial_{i} V_{0} & =H_{i}^{0}-K_{a} H_{i}^{a}+\frac{1}{2}\left|\epsilon^{a b c}\right| K_{a} K_{b} H_{c i}, \\
\partial_{i} K_{a} & =\frac{H_{a}}{2 H_{1} H_{2} H_{3}}\left[\sum_{b \neq a} H_{a} H_{i}^{a}-H_{b} H_{i}^{b}+\left|\epsilon^{b c d}\right| K_{b} H_{c} H_{d i}-2 H_{\substack{a \\
b \neq a \neq c \\
b \neq c}} K_{b} H_{c i}\right], \\
\chi_{i} & =\partial_{i} B-\left|\epsilon^{a b c}\right| \partial_{i} K_{a} H_{b} H_{c},
\end{aligned}
$$

in which $H_{i}^{0}, H_{i}^{a}$ and $H_{a i}=\partial_{i} H_{a}$ are components of the divergenceless symplectic vector $H_{i}^{\alpha}$ introduced in (2.15). Without entering in the details e.g. of the two-centered solution, it should be remarked here that in the composite non-BPS class the fact that $H_{i}^{0}$ and $H_{i}^{a}$ are not harmonic implies the centers to exhibit mutually non-local electric-magnetic fluxes. The same holds for the magnetic solution considered in section 4.2.2.

One can check that this solution satisfies the composite non-BPS first order equations derived above if the phases $\alpha_{a}$ 's are fixed as follows:

$$
\frac{1}{2} \sum_{b \neq a} \alpha_{b}=\pi-\arg z^{a} .
$$


The condition (4.20) is a particular solution to the partial differential equations (4.2), and it actually fixes the two non-BPS "flat" directions, whose presence cannot thus be recognized in the explicit form (4.18)-(4.19) of this solution. Moreover, it should be noted that (4.20) yields the vanishing of the remaining components (not entering (4.19)) of the divergenceless symplectic vector $H_{i}^{\alpha}$ :

$$
H_{0 i}=0 .
$$

Physically, this means that the graviphoton electric charge $q_{0}$ pertaining to each $\mathrm{BH}$ center should vanish. However, in the two-centered case, it can be checked that in general the charge vectors pertaining to each of the two centers are mutually non-local, even if there are no solutions in this class in which all two-centered duality invariants [39] are independent (also cfr. the analysis in [34]).

Multi-centered integrals of motion. Although introduced for single-centered BH solutions, the definition (4.9) of integrals of the $D=3$ motion still holds for multi-centered BHs. In this case, the constraint (4.6) can be rewritten as

$$
\left[\prod_{a} \operatorname{Im} e^{i \beta_{a}}\right]\left\langle H_{i}, V_{\lambda}\right\rangle=0 .
$$

Assuming that the phases $\alpha_{a}$ are such that the product of the imaginary parts (recall definition (4.9)) does not vanish, one obtains that that the skew-symmetric symplectic product $\left\langle H_{i}, V_{\lambda}\right\rangle$ necessarily vanishes:

$$
\left\langle H_{i}, V_{\lambda}\right\rangle=H_{0 i}+H_{a i} \lambda^{a}-3 H_{i}^{(1} \lambda^{2} \lambda^{3)}+H_{i}^{0} \lambda^{1} \lambda^{2} \lambda^{3}=0 .
$$

From the definition of integrals of the $D=3$ motion, and especially from its inverse (4.13), one can see that (4.20) holds iff all integrals of motion equal to zero: $\lambda^{a}=0, \forall a=1,2,3$. In order to fulfill the constraint (4.23), one has to require $H_{0 i}=0$, matching (4.21).

The product of the imaginary parts in (4.22) might vanish. This happens for example in the case of the so-called magnetic configuration, which analysis is performed below.

\subsubsection{Magnetic configuration}

If all phases $\alpha_{a}$ vanish, then the constraint (4.2) turns into an identity. Despite the fact that the definition (4.9) of integrals of motion becomes singular, a careful analysis yields that the constraint (4.22) is satisfied when

$$
H_{i}^{0}=0
$$

Physically, this condition means that the graviphoton magnetic charge $p^{0}$ of each $\mathrm{BH}$ center should vanish. This case was considered in [2]; therein, an explicit solution in the magnetic configuration

$$
e^{-4 U}=4 V_{0} H^{1} H^{2} H^{3}-B^{2}, \quad z^{a}=K^{a}+\frac{H^{a}}{2 H^{1} H^{2} H^{3}}\left(B-i e^{-2 U}\right)
$$


was found, in which the function $V_{0}$ as well as the harmonic functions $K^{a}$ satisfy the first order equations (no summation over index $a$ ):

$$
\begin{aligned}
\partial_{i} V_{0} & =-H_{0 i}-K^{b} H_{b i}+\frac{1}{2}\left|\epsilon_{b c d}\right| K^{b} K^{c} \partial_{i} H^{d}, \\
\partial_{i} K^{a} & =\frac{H^{a}}{2 H^{1} H^{2} H^{3}}\left[H^{a} H_{a i}-\sum_{b \neq a} H^{b} H_{b i}+\left|\epsilon_{b c d}\right| H^{b} K^{c} \partial_{i} H^{d}-2 H^{a} \sum_{\substack{b \neq a \neq c \\
b \neq c}} K^{b} H_{i}^{c}\right] .
\end{aligned}
$$

Thus, the $D=3$ Cartesian rotation vector reads

$$
\chi_{i}=\partial_{i} B+\left|\epsilon_{a b c}\right| \partial_{i} K^{a} H^{b} H^{c}
$$

This solution, which is a particular solution of the composite non-BPS first order equations, is written in terms of the functions $H_{0 i}, H_{a i}$ and $H_{i}^{a}=\partial_{i} H^{a}$, which are components of the divergenceless symplectic vector $H_{i}^{\alpha}$ introduced in (2.15).

\section{Almost BPS class}

For the almost BPS $[1,3]$ class of flows, the first order equations are given by the general eqs. (3.3) supplemented with the flow-defining functions $W_{i}$ and $\Pi_{i}^{a}$ given by eqs. (3.28). Then, one has to satisfy the Einstein constraint (3.7), yielding the following expression for the $D=3$ Cartesian rotation vector $\ell_{i}$ :

$$
\ell_{i}=\frac{1}{4} \operatorname{Im}\left[e^{-i \alpha_{0}}\left(3+e^{i\left(\alpha_{0}+\sum_{a} \alpha_{a}\right)}\right) \hat{Z}_{i}+\left(1-e^{-i\left(\alpha_{0}+\sum_{a} \alpha_{a}\right)}\right) \sum_{a} e^{i \alpha_{a}} \hat{Z}_{a i}\right]
$$

As described in the section 4 , if one requires the equivalence of the first order equations to their second order ancestors, the following four partial differential eqs. for the four phases $\alpha_{0}$ and $\alpha_{a}$ 's are obtained:

$$
\begin{aligned}
\partial_{i} \alpha_{0}= & e^{U} \operatorname{Im}\left[e^{-i \alpha_{0}}\left(\hat{Z}_{i}+\sum_{a} \hat{Z}_{a i}\right)\right] \\
& -\frac{1}{4} e^{U}\left[\left(e^{i \sum_{a} \alpha_{a}}-\sum_{a} e^{i \alpha_{a}}+e^{-i \alpha_{0}}\left(\sum_{a} e^{i \sum_{b \neq a} \alpha_{b}}-1\right)\right) \hat{Z}_{i}\right. \\
& \left.+\sum_{a}\left[\left(e^{i \alpha_{a}}+e^{-i \alpha_{0}}\right)\left(1-e^{-i \sum_{b \neq a} \alpha_{b}}\right)-\sum_{b \neq a} e^{-i \alpha_{b}}\left(1-e^{i \alpha_{a}-i \alpha_{0}}\right)\right] \hat{Z}_{a i}\right], \\
\partial_{i} \alpha_{a}= & -\partial_{i} \alpha_{0}-\frac{1}{2} e^{U} \cos \frac{\left(\alpha_{0}+\alpha_{a}\right)}{2} \operatorname{Im}\left[e ^ { - \frac { i } { 2 } ( \alpha _ { 0 } - \alpha _ { a } ) } \left[\left(e^{i \sum_{b \neq a} \alpha_{b}}-1\right) \hat{Z}_{i}\right.\right. \\
& \left.\left.-\left(e^{-i \sum_{b \neq a} \alpha_{b}}+3\right) \hat{Z}_{a i}\right]+e^{-\frac{i}{2}\left(\alpha_{0}+\alpha_{a}\right)} \sum_{b \neq a}\left(e^{i \alpha_{b}}-e^{-i \alpha_{c}}\right) \hat{Z}_{b i}\right]
\end{aligned}
$$


in which the index $c$ must be different from both $a$ and $b$. In turn, eqs. (5.2) yield a useful relation:

$\partial_{i}\left[\alpha_{0}+\sum_{a} \alpha_{a}\right]=2 e^{U} \operatorname{Re}\left[e^{\frac{i}{2}\left(\alpha_{0}+\sum_{a} \alpha_{a}\right)}\right] \operatorname{Im}\left[e^{\frac{i}{2}\left(\alpha_{0}+\sum_{a} \alpha_{a}\right)}\left(\sum_{a} e^{i \alpha_{a}} \hat{Z}_{a i}-e^{-i\left(\alpha_{0}+\sum_{a} \alpha_{a}\right)} \hat{Z}_{i}\right)\right]$.

In order to integrate the Maxwell eqs. (2.15), we exploit the same approach considered in section 4 ; the flow-defining functions $W_{i}$ and $\chi_{i}$, as well as the electromagnetic potential $b^{\alpha}$, can again be represented as in (4.3) and (4.5) in terms of symplectic vector $T$, which for the class under consideration has a slightly more complicated expression, namely:

$$
T^{\alpha}=-\frac{1}{4}\left(e^{-i \sum_{a} \alpha_{a}} \bar{V}^{\alpha}-3 e^{-i \alpha_{0}} V^{\alpha}+\sum_{a} e^{-i \alpha_{a}} \bar{V}_{\bar{a}}^{\alpha}+e^{-i\left(\alpha_{0}+\sum_{a} \alpha_{a}\right)} \sum_{a} e^{i \alpha_{a}} V_{a}^{\alpha}\right) .
$$

When replacing the electromagnetic potential $b$ expressed in terms of $T$ (5.4) into eq. (5.1), this latter is turned into a constrant:

$$
\operatorname{Im}\left[e^{-i \alpha_{0}}\left(3+e^{i\left(\alpha_{0}+\sum_{a} \alpha_{a}\right)}\right) Z_{i}+\left(1-e^{-i\left(\alpha_{0}+\sum_{a} \alpha_{a}\right)}\right) \sum_{a} e^{i \alpha_{a}} Z_{a i}\right]=0 .
$$

In other words, as for the composite non-BPS class treated in section 4, if one tries to deduce $\chi_{i}$ from (5.1) recalling that (cfr. (2.15) and (2.24))

$$
\hat{Z}_{i}=Z_{i}+\chi_{i}\langle b, V\rangle, \quad \hat{Z}_{a i}=Z_{a i}+\chi_{i}\left\langle b, V_{a}\right\rangle
$$

then it can be checked that the dependence of (5.1) on $\chi_{i}$ drops out, thus yielding the constraint (5.5).

However, differently from the composite non-BPS class, in the almost BPS class, the Maxwell equations turn out to be consistent only when two further constraints are satisfied, namely:

$$
\mathbf{I}: \alpha_{0}+\sum_{a} \alpha_{a}=\pi \quad \text { or } \quad \mathbf{I I}: \alpha_{0}+\sum_{a} \alpha_{a}=2 \arctan \left(e^{2 U} B\right),
$$

thus giving rise, as in the $t^{3}$ model investigated in [25], to two distinct almost BPS branches, ${ }^{8}$ which we will separately analyzed in the next two subsections.

The counting of the two "flat" directions along the (non-BPS, "large") flows in the stu model $[23,35]$ is retrieved by considering that in this class the four partial differential eqs. (5.2) for the four phases $\alpha_{0}$ and $\alpha_{a}$ 's are supplemented by the algebraic constraint (5.5) and by the further condition I or II of (5.7).

\footnotetext{
${ }^{8}$ It is interesting to notice that each branch admits a single-centered limit of different type: while branch I admits a particular BPS BH with $i_{3}=0$ (cfr. section 5.1), branch II exhibits a non-BPS BH (as singlecentered limit of the particular set of embedded composite non-BPS solutions discussed in section 5.2). This is consistent with the fact that two-centered almost BPS solutions are characterized by a BPS and a non-BPS center $[1,34]$.
} 


\subsection{Branch I}

In the case $\mathbf{I}$ of (5.7), the $D=3$ Cartesian rotation vector reads

$$
\chi_{i}=\partial_{i} B+e^{U} B \operatorname{Re}\left[\sum_{a} e^{i \alpha_{a}} Z_{a i}+3 e^{-i \alpha_{0}} Z_{i}\right]+e^{-U} \operatorname{Im}\left[\sum_{a} e^{i \alpha_{a}} Z_{a i}-e^{-i \alpha_{0}} Z_{i}\right],
$$

and expressions for the flow-defining functions gets slightly simplified as follows:

$$
\begin{aligned}
& W_{i}=\operatorname{Re}\left[e^{-i \alpha_{0}} \hat{Z}_{i}\right], \\
& \Pi_{i}^{a}=e^{i \alpha_{0}} \hat{Z}_{a i}+\frac{i}{2} e^{i\left(\alpha_{0}+\alpha_{a}\right)} \operatorname{Im}\left(e^{i \sum_{b} \alpha_{b}} \hat{Z}_{i}+\sum_{b} e^{i \alpha_{b}} \hat{Z}_{b i}\right) .
\end{aligned}
$$

This branch contains BPS multi-centered solutions [16] with a particular constraint. This can be proved by simply setting $B=0$ in (5.8), thus implying the functions $W_{i}$ and $\Pi_{i}^{a}$ to turn into their BPS counterparts (3.15), whit rotation $\chi_{i}$ acquiring the following form:

$$
\chi_{i}=e^{-U} \operatorname{Im}\left[e^{i \sum_{a} \alpha_{a}} Z_{i}+\sum_{a} e^{i \alpha_{a}} Z_{i a}\right] .
$$

Independently on the value of the $B$-field, the constraint (5.5) takes the form

$$
\operatorname{Im}\left[e^{i \sum_{a} \alpha_{a}} Z_{i}-\sum_{a} e^{i \alpha_{a}} Z_{i a}\right]=0
$$

Thus, (5.10) and (5.11) yield

$$
\chi_{i}=-2 e^{-U} \operatorname{Im}\left[e^{-i \alpha_{0}} Z_{i}\right]=2 e^{-U} \operatorname{Im}\left[\sum_{a} e^{i \alpha_{a}} Z_{a i}\right],
$$

which is exactly the expression of the $D=3$ rotation pertaining to the BPS multi-centered class [16] subjected to the additional constraint (5.11).

The actual meaning of the constraint (5.11) has yet to be clarified; nevertheless, we can here make some observations. Considering the corresponding single-centered case, and thus setting both $B$ and $\chi_{i}$ to zero, this constraint splits in two parts, namely:

$$
\operatorname{Im}\left[\sum_{a} e^{i \alpha_{a}} Z_{a i}\right]=0 \quad \text { and } \quad \operatorname{Im}\left[e^{i \sum_{a} \alpha_{a}} Z_{i}\right]=0
$$

In this case, at least a particular solution

$$
\alpha_{a}=-\arg Z_{a}, \quad \arg Z=\sum_{a} \arg Z_{a}
$$

of the constraint $\mathbf{I}$ of (5.7) can be shown to be consistent with the first order equations and, as a consequence, the duality invariant $i_{3}[24,40]$ vanishes. In this sense, the constraint (5.11) can be regarded as the generalization, within the BPS multi-centered flow embedded into the branch $\mathbf{I}$ of the almost BPS class, of the constraint $i_{3}=0$. 
It should be stressed that the ADM mass of this branch is bounded from above by the BPS mass. This can be easily realized by rewriting the asymptotical limit of expressions (5.9) using the constraint (5.5) (or equivalently (5.11)) as well as the branch $\mathbf{I}$ condition (5.7), as

$$
W=\operatorname{Re}\left(e^{-i \alpha_{0}} Z\right), \quad \Pi^{a}=e^{i \alpha_{0}} \bar{Z}_{a}-i e^{i\left(\alpha_{0}+\alpha_{a}\right)} \operatorname{Im}\left(e^{-i \alpha_{0}} Z\right) .
$$

By denoting the phase of $Z$ by $\alpha_{Z}$, it thus follows that asymptotically

$$
W=\operatorname{Re}\left(e^{-i \alpha_{0}} Z\right)=\cos \left(\alpha_{Z}-\alpha_{0}\right)|Z| \leqslant|Z|,
$$

as mentioned above. Thus, asymptotically, the ADM mass of the almost-BPS branch I either violates the BPS bound (thus giving rise to a naked singularity, according to the weakest version of the cosmic censorship [41]), or saturates it. This latter case implies the solution to preserve asymptotically one half of the supersymmetries.

At the moment, it is however not known whether this necessarily extends all along the flow, namely whether this branch contains other consistent multi-centered solutions different from the aforementioned (constrained) BPS solutions (especially if one of the centers is "small"); we leave this issue for further investigation.

\subsection{Branch II}

In the case II of (5.7), the $D=3$ Cartesian rotation vector reads

$$
\chi_{i}=\partial_{i} B-B e^{U} \operatorname{Re}\left[3 e^{i \sum_{a} \alpha_{a}} Z_{i}-\sum_{a} e^{i \alpha_{a}} Z_{a i}\right]+e^{-U} \operatorname{Im}\left[3 e^{i \sum_{a} \alpha_{a}} Z_{i}-\sum_{a} e^{i \alpha_{a}} Z_{a i}\right],
$$

and the constraint (5.5) acquires the following form:

$$
\begin{aligned}
& 2 \operatorname{Im}\left[e^{i \sum_{a} \alpha_{a}} Z_{i}\right]-B e^{2 U} \operatorname{Re}\left[3 e^{i \sum_{a} \alpha_{a}} Z_{i}-\sum_{a} e^{i \alpha_{a}} Z_{a i}\right] \\
&-B^{2} e^{4 U} \operatorname{Im}\left[e^{i \sum_{a} \alpha_{a}} Z_{i}-\sum_{a} e^{i \alpha_{a}} Z_{a i}\right]=0 .
\end{aligned}
$$

Here we refrain from writing down the explicit expressions for the flow-defining functions $W_{i}$ and $\Pi_{i}^{a}$, resulting from plugging eq. (5.7) (case II) into (3.28), because in this branch their form is not very illuminating and rather cumbersome.

It is worth remarking that, among all possible solutions to eqs. (5.17)-(5.18), there is a particular one that coincides with a particular solution of the composite non-BPS treated in section 5 ; indeed, if the $B$-field is set to a constant and the rotation $\chi_{i}$ vanishes, then it can be checked that the set of equations (5.17)-(5.18) turns into the set of eqs. (4.6) and (4.7), which describes the composite non-BPS class.

An example with fixed "flat" directions. An interesting example is provided by the choice of all phases $\alpha_{a}$ 's to be equal. The consistency of eqs. (5.2) within the case II of (5.7) requires then that

$$
\alpha_{1}=\alpha_{2}=\alpha_{3}=-\frac{\pi}{2}+\arctan B e^{2 U}, \quad \alpha_{0}=\frac{3 \pi}{2}-\arctan B e^{2 U} .
$$


This latter exactly falls into the class of solutions $[1,3]$

$$
e^{-4 U}=4 H^{0} \mathcal{Z}_{1} \mathcal{Z}_{2} \mathcal{Z}_{3}-B^{2}, \quad z^{a}=K^{a}-\frac{B-i e^{2 U}}{2 H^{0} \mathcal{Z}_{a}}
$$

where $H^{0}$ is harmonic, and the functions $\mathcal{Z}_{a}$ and $K^{a}$ satisfy the following equations:

$$
\partial_{i} \mathcal{Z}_{a}=H_{i a}-\left|\epsilon_{a b c}\right|\left(H_{i}^{b}-\frac{1}{2} K^{b} \partial_{i} H^{0}\right) K^{c}, \quad \partial_{i} K_{a}=-\frac{1}{H^{0}}\left(H_{i}^{a}-K^{a} \partial_{i} H^{0}\right),
$$

where the latter equation implies $K^{a}$ to be harmonic. In this case, the $D=3$ Cartesian rotation vector $\chi_{i}$ can be rewritten as

$$
\chi_{i}=\partial_{i} B-2 H^{0} \partial_{i} K^{a} \mathcal{Z}_{a},
$$

and the constraint (5.5) reads

$$
H_{i 0}+H_{i a} K^{a}+\frac{1}{2}\left|\epsilon_{a b c}\right| \partial_{i} K^{a} K^{b} K^{c} H^{0}-\partial_{i} K^{a} \mathcal{Z}_{a}-2 K^{1} K^{2} K^{3} \partial_{i} H^{0}=0 .
$$

This solution can generate other solutions by applying the $U$-duality and Ehlers symmetry transformations, as considered in [1]. We leave to further future investigation the issue of existence of general multi-centered solutions of this class; for instance, in the twocentered case, such general solutions should exhibit all the duality-invariant polynomials of the "minimal degree" complete basis [39] as independent.

\section{Conclusion}

In the present investigation, we developed the most general first order formalism for multicentered and/or under-rotating extremal $\mathrm{BH}$ solutions with flat three-dimensional basespace in the stu model $[23,27,28]$ of $N=2, D=4$ ungauged Maxwell-Einstein supergravity. As mentioned, this is a universal sector of all $N \geqslant 2$-extended supergravity theories with symmetric (vector multiplets') scalar manifold, and which admit an uplift to $D=5$ dimensions.

Our procedure, which generalizes the one exploited for the simpler $t^{3}$ model in [25], sets the non-supersymmetric (composite non-BPS [1] and almost BPS [1,3]) classes of multi-centered solutions on the same footing of the well known BPS class [16, 17], thus allowing for a unified framework for the study of the flow dynamics of scalar fields.

We developed a tree-dimensional Cartesian formalism in which the effective BH potential [30], as well as the scalar-dependent central and matter charges and the first order "fake" superpotential [18], are generalized in a not necessarily axisymmetric framework (cfr. (2.18), (2.24), (2.25) and (3.4)), which thus allows to handle also (under-)rotating and multi-centered $\mathrm{BH}$ solutions.

Then, by extending some spatially asymptotical expressions all along the flow, we derived systems of partial differential equations for the $\alpha$-phases describing each class of multi-centered systems. The corresponding counting, supplemented by an algebraic constraint (and by a further condition in the almost-BPS class) is consistent with the existence of two "flat" directions along the non-rotating sigle-centered stu non-BPS flow [23, 35]. 
The consistency of the systems of partial differential equations on which the first order formalism is based has then been checked also by retrieving known solutions, in the single-centered limit (such as the Rasheed-Larsen non-BPS Kaluza-Klein BH [37, 38] in the composite non-BPS class) as well as in the multi-centered case (various solutions from $[1,2,17]$ have been obtained as particular solutions).

Consistent with its nilpotent orbit characterization [1] as well as with the results obtained in [25] for the $t^{3}$ model, the almost BPS class [1,3] exhibits the most involved structure: indeed, it is described by four independent phases $\alpha_{0}$ and $\alpha_{a}(a=1,2,3)$, and in this class the consistency of Maxwell equations further imposes the constraint (5.7), whose two-fold nature gives rise to two sub-branches. Such a split can be traced back to the possibility to have both BPS and non-BPS BH centers in this class, which thus stands on a different footing with respect to the BPS and composite non-BPS classes, in which the $\mathrm{BH}$ centers are of the same type.

As mentioned in sections 4 and 5 , we leave for future investigation the issue of existence of completely general solutions (with all electric-magnetic charges switched on in each $\mathrm{BH}$ center) in the composite non-BPS and/or almost BPS classes. In the two-centered case, such general solutions would exhibit all independent duality-invariant polynomials of the complete "minimal degree" basis $[34,39,44]$. Moreover, it would be interesting to analyze the issue of the fixed distance among the $\mathrm{BH}$ centers in the non-supersymmetric systems $[1,2,4,5]$, also in relation to the existence of walls of marginal stability.

It is here worth pointing out that we considered only multi-centered configurations with "large" BH centers, namely with a well-defined near-horizon geometry (and thus, scalar dynamics) already at the Einstein (two-derivatives) level; at the moment, it is not clear whether some solutions with "small" BH centers (cfr. e.g. [13, 45]) can be obtained in this framework (for an analysis in the two-centered case at the level of duality invariants, see [34]).

The possible extension of our approach to multi-centered systems with non-flat threedimensional base-space is of utmost interest, as well; within this framework, one should recover solutions found e.g. in $[4,5]$. It would also be interesting to consider generalizations to over-rotating (single- and multi- centered) BH solutions (for recent advances on first order formalism, see e.g. [46]).

Our approach could also be applied to the multi-centered interacting non-BPS solutions of maximal $N=8, D=4$ supergravity recently considered e.g. in [2], and the issue of truncability to the universal stu sector is also worth being investigated in greater detail.

\section{Acknowledgments}

We would like to thank G. Bossard, A. Ceresole and G. Dall'Agata for useful discussions and correspondence.

The work of S.F. and A. Y. has been supported by the ERC Advanced Grant no. 226455, Supersymmetry, Quantum Gravity and Gauge Fields (SUPERFIELDS). 


\section{A Notations and useful formulæ}

We here recall some useful notations and formulæ, used throughout the present investigation.

In a generic special Kähler geometry, the symmetric real matrix $M$, which firstly occurred in our treatment in eq. (2.7), is constructed from the coupling matrices $\mu_{\Lambda \Sigma}$ and $\nu_{\Lambda \Sigma}$ as follows $[31,42,43]$

$$
M_{\alpha \beta}=\left(\begin{array}{cc}
\mu_{\Lambda \Sigma}+\nu_{\Lambda \Lambda^{\prime}} \mu^{\Lambda^{\prime} \Sigma^{\prime}} \nu_{\Sigma^{\prime} \Sigma} & \nu_{\Lambda \Lambda^{\prime}} \mu^{\Lambda^{\prime} \Sigma} \\
\mu^{\Lambda \Lambda^{\prime}} \nu_{\Lambda^{\prime} \Sigma} & \mu^{\Lambda \Sigma}
\end{array}\right),
$$

and it is symplectic:

$$
\Omega^{\alpha \beta} M_{\beta \gamma}=-M^{\alpha \beta} \Omega_{\beta \gamma}
$$

with respect to the skew-symmetric symplectic metric

$$
\Omega_{\alpha \beta}=\left(\begin{array}{cc}
0 & -\delta_{\Lambda}^{\Sigma} \\
\delta_{\Sigma}^{\Lambda} & 0
\end{array}\right), \quad \Omega^{\alpha \beta}=\left(\begin{array}{cc}
0 & \delta_{\Sigma}^{\Lambda} \\
-\delta_{\Lambda}^{\Sigma} & 0
\end{array}\right),
$$

which allows one to define the symplectic product of two vectors as:

$$
\langle A, B\rangle \equiv A^{\alpha} \Omega_{\alpha \beta} B^{\beta}=A_{\Lambda} B^{\Lambda}-A^{\Lambda} B_{\Lambda} .
$$

The inverse of the coupling matrix can be easily calculated to read

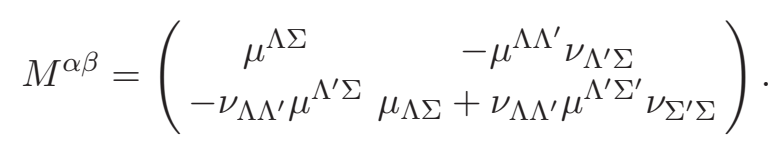

The special geometry sections are normalized as

$$
\langle V, \bar{V}\rangle=-i, \quad\left\langle V, D_{a} V\right\rangle=0, \quad\left\langle D_{a} V, \bar{D}_{\bar{a}} \bar{V}\right\rangle=i G_{a \bar{a}} .
$$

The coupling matrix $M$ satisfy the following identity

$$
\frac{1}{2}\left(M_{\alpha \beta}-i \Omega_{\alpha \beta}\right)=-\left(\Omega_{\alpha \alpha^{\prime}} \bar{V}^{\alpha^{\prime}}\right)\left(\Omega_{\beta \beta^{\prime}} V^{\beta^{\prime}}\right)-\left(\Omega_{\alpha \alpha^{\prime}} D_{a} V^{\alpha}\right) G^{a \bar{a}}\left(\Omega_{\beta \beta^{\prime}} \bar{D}_{\bar{a}} \bar{V}^{\beta^{\prime}}\right) .
$$

The $C$-tensor of special Kähler geometry and the metric of the vector multiplets' scalar manifold enter the basic relations

$$
D_{a} D_{b} V=i C_{a b c} G^{c \bar{c}} \bar{D}_{\bar{c}} \bar{V}, \quad D_{a} \bar{D}_{\bar{b}} \bar{V}=G_{a \bar{b}} \bar{V} .
$$

In the $s t u$ model

$$
F=D_{a b c} z^{a} z^{b} z^{c}=s t u,
$$

the Vielbein and its inverse (we maintain here the underlining of flat scalar indices)

$$
E_{a} \underline{a}=\operatorname{diag}\left(\frac{1}{s-\bar{s}}, \frac{1}{t-\bar{t}}, \frac{1}{u-\bar{u}}\right), \quad E_{\underline{a}}{ }^{a}=\operatorname{diag}(s-\bar{s}, t-\bar{t}, u-\bar{u})
$$

satisfy the usual definition

$$
G_{a \bar{a}}=E_{a} \underline{a} \delta_{\underline{a} \bar{a}} \bar{E}_{\bar{a}} \overline{\bar{a}}^{\underline{a}}, \quad \delta_{\underline{a}} \underline{\bar{a}}=\operatorname{diag}(1,1,1) .
$$


Open Access. This article is distributed under the terms of the Creative Commons Attribution License which permits any use, distribution and reproduction in any medium, provided the original author(s) and source are credited.

\section{References}

[1] G. Bossard and C. Ruef, Interacting non-BPS black holes, Gen. Rel. Grav. 44 (2012) 21 [arXiv: 1106.5806] [INSPIRE].

[2] G. Bossard, Octonionic black holes, JHEP 05 (2012) 113 [arXiv:1203.0530] [INSPIRE].

[3] K. Goldstein and S. Katmadas, Almost BPS black holes, JHEP 05 (2009) 058 [arXiv:0812.4183] [INSPIRE].

[4] I. Bena, G. Dall'Agata, S. Giusto, C. Ruef and N.P. Warner, Non-BPS Black Rings and Black Holes in Taub-NUT, JHEP 06 (2009) 015 [arXiv:0902.4526] [INSPIRE].

[5] I. Bena, S. Giusto, C. Ruef and N.P. Warner, Multi-Center non-BPS Black Holes: the Solution, JHEP 11 (2009) 032 [arXiv:0908.2121] [INSPIRE].

[6] I. Bena, S. Giusto, C. Ruef and N.P. Warner, Supergravity Solutions from Floating Branes, JHEP 03 (2010) 047 [arXiv:0910.1860] [INSPIRE].

[7] G. Dall'Agata, S. Giusto and C. Ruef, U-duality and non-BPS solutions, JHEP 02 (2011) 074 [arXiv: 1012.4803] [INSPIRE].

[8] I. Bena, S. Giusto and C. Ruef, A Black Ring with two Angular Momenta in Taub-NUT, JHEP 06 (2011) 140 [arXiv:1104.0016] [INSPIRE].

[9] P. Fré and A.S. Sorin, Extremal Multicenter Black Holes: Nilpotent Orbits and Tits Satake Universality Classes, JHEP 01 (2013) 003 [arXiv:1205.1233] [INSPIRE].

[10] D. Gaiotto, W. Li and M. Padi, Non-Supersymmetric Attractor Flow in Symmetric Spaces, JHEP 12 (2007) 093 [arXiv:0710.1638] [INSPIRE].

[11] T. Ortín and C. Shahbazi, The supersymmetric black holes of $N=8$ supergravity, Phys. Rev. D 86 (2012) 061702 [arXiv:1206.3190] [INSPIRE].

[12] G. Bossard and S. Katmadas, Duality covariant non-BPS first order systems, JHEP 09 (2012) 100 [arXiv:1205.5461] [INSPIRE].

[13] E.G. Gimon, F. Larsen and J. Simon, Constituent Model of Extremal non-BPS Black Holes, JHEP 07 (2009) 052 [arXiv:0903.0719] [INSPIRE].

[14] P. Galli and J. Perz, Non-supersymmetric extremal multicenter black holes with superpotentials, JHEP 02 (2010) 102 [arXiv:0909.5185] [INSPIRE].

[15] P. Galli, K. Goldstein, S. Katmadas and J. Perz, First-order flows and stabilisation equations for non-BPS extremal black holes, JHEP 06 (2011) 070 [arXiv:1012.4020] [INSPIRE].

[16] F. Denef, Supergravity flows and D-brane stability, JHEP 08 (2000) 050 [hep-th/0005049] [INSPIRE].

[17] B. Bates and F. Denef, Exact solutions for supersymmetric stationary black hole composites, JHEP 11 (2011) 127 [hep-th/0304094] [inSPIRE].

[18] A. Ceresole and G. Dall'Agata, Flow Equations for Non-BPS Extremal Black Holes, JHEP 03 (2007) 110 [hep-th/0702088] [INSPIRE]. 
[19] L. Andrianopoli, R. D'Auria, E. Orazi and M. Trigiante, First order description of black holes in moduli space, JHEP 11 (2007) 032 [arXiv:0706.0712] [INSPIRE].

[20] L. Andrianopoli, R. D'Auria, E. Orazi and M. Trigiante, First Order Description of D $=4$ static Black Holes and the Hamilton-Jacobi equation, Nucl. Phys. B 833 (2010) 1 [arXiv:0905.3938] [INSPIRE].

[21] G. Bossard, Y. Michel and B. Pioline, Extremal black holes, nilpotent orbits and the true fake superpotential, JHEP 01 (2010) 038 [arXiv:0908.1742] [INSPIRE].

[22] A. Ceresole, G. Dall'Agata, S. Ferrara and A. Yeranyan, Universality of the superpotential for D $=4$ extremal black holes, Nucl. Phys. B 832 (2010) 358 [arXiv:0910.2697] [InSPIRE].

[23] S. Bellucci, S. Ferrara, A. Marrani and A. Yeranyan, stu Black Holes Unveiled, Entropy 10 (2008) 507 [arXiv: 0807.3503] [INSPIRE].

[24] A. Ceresole, G. Dall'Agata, S. Ferrara and A. Yeranyan, First order flows for $N=2$ extremal black holes and duality invariants, Nucl. Phys. B 824 (2010) 239 [arXiv:0908.1110] [INSPIRE].

[25] A. Yeranyan, Multi-Centered Black Hole Flows, JHEP 08 (2012) 158 [arXiv:1205.5618] [INSPIRE].

[26] B. de Wit, F. Vanderseypen and A. Van Proeyen, Symmetry structure of special geometries, Nucl. Phys. B 400 (1993) 463 [hep-th/9210068] [inSPIRE].

[27] M. Duff, J.T. Liu and J. Rahmfeld, Four-dimensional string-string-string triality, Nucl. Phys. B 459 (1996) 125 [hep-th/9508094] [INSPIRE].

[28] K. Behrndt, R. Kallosh, J. Rahmfeld, M. Shmakova and W.K. Wong, STU black holes and string triality, Phys. Rev. D 54 (1996) 6293 [hep-th/9608059] [INSPIRE].

[29] P. Breitenlohner, D. Maison and G.W. Gibbons, Four-Dimensional Black Holes from Kaluza-Klein Theories, Commun. Math. Phys. 120 (1988) 295 [InSPIRE].

[30] S. Ferrara, G.W. Gibbons and R. Kallosh, Black holes and critical points in moduli space, Nucl. Phys. B 500 (1997) 75 [hep-th/9702103] [INSPIRE].

[31] A. Ceresole, R. D'Auria and S. Ferrara, The symplectic structure of $N=2$ supergravity and its central extension, Nucl. Phys. Proc. Suppl. 46 (1996) 67 [hep-th/9509160] [InSPIRE].

[32] A. Strominger, Special geometry, Commun. Math. Phys. 133 (1990) 163 [INSPIRE].

[33] L. Andrianopoli et al., $N=2$ supergravity and $N=2$ super Yang-Mills theory on general scalar manifolds: Symplectic covariance, gaugings and the momentum map,

J. Geom. Phys. 23 (1997) 111 [hep-th/9605032] [INSPIRE].

[34] A. Ceresole, S. Ferrara, A. Marrani and A. Yeranyan, Small Black Hole Constituents and Horizontal Symmetry, JHEP 06 (2011) 078 [arXiv:1104.4652] [INSPIRE].

[35] S. Ferrara and A. Marrani, On the Moduli Space of non-BPS Attractors for $N=2$ Symmetric Manifolds, Phys. Lett. B 652 (2007) 111 [arXiv:0706.1667] [INSPIRE].

[36] E.G. Gimon, F. Larsen and J. Simon, Black holes in Supergravity: The Non-BPS branch, JHEP 01 (2008) 040 [arXiv:0710.4967] [INSPIRE].

[37] D. Rasheed, The rotating dyonic black holes of Kaluza-Klein theory, Nucl. Phys. B 454 (1995) 379 [hep-th/9505038] [INSPIRE]. 
[38] F. Larsen, Rotating Kaluza-Klein black holes, Nucl. Phys. B 575 (2000) 211 [hep-th/9909102] [INSPIRE].

[39] S. Ferrara, A. Marrani, E. Orazi, R. Stora and A. Yeranyan, Two-Center Black Holes Duality-Invariants for STU Model and its lower-rank Descendants, J. Math. Phys. 52 (2011) 062302 [arXiv:1011.5864] [INSPIRE].

[40] B.L. Cerchiai, S. Ferrara, A. Marrani and B. Zumino, Duality, Entropy and ADM Mass in Supergravity, Phys. Rev. D 79 (2009) 125010 [arXiv:0902.3973] [INSPIRE].

[41] R. Kallosh, A.D. Linde, T. Ortín, A.W. Peet and A. Van Proeyen, Supersymmetry as a cosmic censor, Phys. Rev. D 46 (1992) 5278 [hep-th/9205027] [INSPIRE].

[42] S. Ferrara and R. Kallosh, Supersymmetry and attractors, Phys. Rev. D 54 (1996) 1514 [hep-th/9602136] [INSPIRE].

[43] S. Ferrara and R. Kallosh, Universality of supersymmetric attractors, Phys. Rev. D 54 (1996) 1525 [hep-th/9603090] [INSPIRE].

[44] S. Ferrara, A. Marrani and A. Yeranyan, On Invariant Structures of Black Hole Charges, JHEP 02 (2012) 071 [arXiv:1110.4004] [INSPIRE].

[45] A. Castro and J. Simon, Deconstructing the D0-D6 system, JHEP 05 (2009) 078 [arXiv: 0903.5523] [INSPIRE].

[46] L. Andrianopoli, R. D'Auria, P. Giaccone and M. Trigiante, Rotating black holes, global symmetry and first order formalism, JHEP 12 (2012) 078 [arXiv:1210.4047] [INSPIRE]. 